\title{
Overexpression of the $\beta$ Subunit of Human Chorionic Gonadotropin Promotes the Transformation of Human Ovarian Epithelial Cells and Ovarian Tumorigenesis
}

\author{
Xiaoqing Guo, ${ }^{* \dagger}$ Guangzhi Liu, ${ }^{\dagger}$ \\ Isaiah G. Schauer, ${ }^{\dagger}$ Gong Yang, ${ }^{\dagger}$ \\ Imelda Mercado-Uribe, ${ }^{\dagger}$ Fan Yang, ${ }^{\dagger}$ \\ Shiwu Zhang, ${ }^{\dagger}$ Yuanli $\mathrm{He},{ }^{*}$ and Jinsong $\mathrm{Liu}^{\dagger}$ \\ From the Department of Obstetrics and Gynecology,* Zhujiang \\ Hospital, Southern Medical University, Guangzhou, China; the \\ Department of Pathology, ${ }^{\dagger}$ The University of Texas M.D. \\ Anderson Cancer Center, Houston, Texas
}

Ovarian carcinoma is the most lethal gynecologic malignancy, however underlying molecular events remain elusive. Expression of human chorionic gonadotropin $\beta$ subunit ( $\beta$-hCG) is clinically significant for both trophoblastic and nontrophoblastic cancers; however, whether $\beta$-hCG facilitates ovarian epithelial cell tumorigenic potential remains uncharacterized. Immortalized nontumorigenic ovarian epithelial T29 and T80 cells stably overexpressing $\beta$-hCG were examined for alterations in cell cycle and apoptotic status by flow cytometry, expression of proteins regulating cell cycle and apoptosis by Western blot, proliferation status by MTT assay, anchorage-independent colony formation, and mouse tumor formation. Immunoreactivity for $\boldsymbol{\beta}$-hCG was evaluated using mouse xenografts and on human normal ovarian, fallopian tube, endometrium, and ovarian carcinoma tissues. T29 and T80 cells overexpressing $\boldsymbol{\beta}$-hCG demonstrated significantly increased proliferation, anchorage-independent colony formation, prosurvival Bcl-X $X_{L}$ protein expression, G2-checkpoint progression, elevated cyclins $\mathrm{E} / \mathrm{D} 1$ and $\mathrm{Cdk} 2 / 4 / 6$, and decreased apoptosis. Collectively, these transformational alterations in phenotype facilitated increased xenograft tumorigenesis $(P<0.05)$. Furthermore, $\beta$-hCG immunoreactivity was elevated in malignant ovarian tumors, compared with normal epithelial expression in ovaries, fallopian tube, and endometrium $(P<0.001)$. Our data indicate that elevated $\beta$-hCG transforms ovarian surface epithelial cells, facilitating proliferation, cell cycle progression, and attenuated apoptosis to promote tumor- igenesis. Our results further decipher the functional role and molecular mechanism of $\boldsymbol{\beta}$-hCG in ovarian carcinoma. $\beta$-hCG may contribute to ovarian cancer etiology, which introduces a new therapeutic intervention target for ovarian cancer. (Am J Pathol 2011, 179:1385-1393; DOI: 10.1016/j.ajpath.2011.05.018)

Ovarian cancer is the most lethal form of gynecologic cancer in the United States, accounting for an estimated 21,550 new cases and 14,600 deaths in 2009. ${ }^{1}$ Survival rates can approach $90 \%$ when ovarian cancer is diagnosed at an early stage; however, early detection is challenging, because the relatively nonspecific symptoms of ovarian lesions may be overlooked until abdominal distension by ascites fluid or by large tumor masses becomes unmistakable. Even with extensive surgical debulking and aggressive chemotherapy, the prognosis for women with ovarian cancer currently is not hopeful. Several studies have indicated that different histological subtypes of ovarian carcinoma are associated with different

Supported by 5R01 CA131183 from the NIH National Cancer Institute and Ovarian Cancer Research Fund (JL). We also acknowledge the support of the MD Anderson SPORE in Ovarian Cancer 5P50 CA83639 and the CCSG shared resources funded, in part, by CA 5 P30CA016672. XG is supported in part by a fellowship from China Natural Science Foundation.

Accepted for publication May 9, 2011.

X.G., G.L., and I.G.S. contributed equally to the present work.

X.G., G.L., and F.Y. are visiting Professors from their affiliated institutes at The U.T. M.D. Anderson Cancer Center.

Current address of X.G.: Department of Obstetrics and Gynecology, Shihezi University School of Medicine, Shihezi, Xinjiang, China; of G.L.: Department of Obstetrics and Gynecology, Henan Provincial People's Hospital, Zhengzhou, Henan, China; of G.Y.: Cancer Research Laboratory, Fudan University Shanghai Cancer Center, Shanghai, Shanghai, China; F.Y.: Department of Pathology, West China Second University Hospital, Sichuan University, Chengdu, Sichuan, China.

Address reprint requests to Jinsong Liu, M.D., Ph.D., Department of Pathology, Unit 85, The University of Texas M.D. Anderson Cancer Center, 1515 Holcombe Blvd., Houston, TX 77030. E-mail: jliu@mdanderson.org. 
causes and underlying mechanisms, including gene amplification, genetic predisposition, and various carcinogens. ${ }^{2-5}$ Nonetheless, the origin and causes of ovarian carcinoma remain to be elucidated.

Human chorionic gonadotropin ( $\mathrm{hCG}$ ) has a physiologically significant role during pregnancy. It is produced as a heterodimeric glycoprotein complex by the placenta over the course of the first 3 months of gestation. The heterocomplex consists of an $\alpha$ subunit and a hormonespecific $\beta$ subunit, which collectively act as a ligand in activating the luteinizing hormone/hCG receptor $(\mathrm{LH} /$ hCGR) in gonadal cells to regulate sex hormone synthesis and reproductive processes. ${ }^{6}$ The $\beta$ subunit of the hCG complex ( $\beta$-hCG) is an accurate marker for diagnosis and monitoring of trophoblastic tumors and ovarian germ cell tumors. ${ }^{7,8}$ Recently, it was shown that elevation in levels of $\beta$-hCG in serum, urine, or tumor tissue correlates with patient outcome in a variety of nontrophoblastic tumors of diverse primary tissue origin. ${ }^{9-15}$ Moreover, elevated $\beta$-hCG was associated with aggressive disease, poor prognosis, and predicted resistance to therapy in bladder cancer patients. ${ }^{16}$ Additionally, increased $\beta$-hCG expression simultaneously stimulates proliferation and inhibits apoptosis of cancer cells derived from the bladder and the cervix in vitro. ${ }^{17,18}$ Furthermore, ovarian carcinoma tissue displays an overexpression not only of the hormone-specific $\beta$-hCG subunit, but also the cognate receptor hCGR. ${ }^{9,19}$ The functional role and molecular mechanism of $\beta$-hCG within ovarian cancer tumorigenesis have yet to be characterized. Recent evidence has strongly implicated epithelial cells derived from the fallopian tube, especially the fimbriated ends, as the likely origin for high-grade serous carcinoma. ${ }^{20}$ To test whether this molecule plays a direct role in facilitating ovarian epithelial cell activation and tumorigenic potential, we designed a panel of experiments using both in vitro and in vivo methods, including evaluation of $\beta$-hCG expression in the normal fallopian tube.

\section{Materials and Methods}

\section{$\beta$-hCG Cloning, Retrovirus Production, and Establishment of Stably Expressing Cells}

To generate human $\beta$-hCG cDNA, total RNA was isolated from placental tissue using TRIzol (Invitrogen, Carlsbad, CA). Reverse transcription with random hexamer primers was performed with an Omniscript RT kit (Qiagen, Valen$\mathrm{cia}, \mathrm{CA}$ ). The sequence for the primers used in amplifying $\beta$-hCG 498bp cDNA was 5'-ACTAGGATCCATGGAGATGTTCCAGGGGCTGCTG-3' and 5'-TGAGAATTCTTATTGTGGGAGGATCGGGGTGTC-3'. Amplified $\beta$-hCG cDNA was digested with BamHI and EcoRI and was ligated into the pBabe-puromycin retrovirus production plasmid. Positive clones were selected by restriction digest confirmation and were verified by DNA sequencing. Retroviruses carrying $\beta$-hCG cDNA were generated and harvested as described previously. ${ }^{21}$ T29 and T80 cells were derived from normal, immortalized nontumorigenic ovarian surface epithelial cell lines IOSE-29 and IOSE-80 expressing human telomerase catalytic subunit by transfection of SV40 T/t antigens and were confirmed as nontumorigenic, as described previously. ${ }^{21}$ T29 $\beta$-hCG and T80 $\beta$-hCG cell lines were generated by double retrovirus infection and stable selection with puromycin $(100 \mu \mathrm{g} /$ $\mathrm{mL}$, Sigma, St. Louis, MO) for 10 to 14 days.

\section{Western Blot}

To confirm $\beta$-hCG overexpression and to analyze the expression of proteins regulating apoptosis and cell cycle progression, cell lysates from T29, T80, T29 $\beta$-hCG, and T80 $\beta$-hCG cell lines were prepared using radioimmunoprecipitation assay buffer $(50 \mathrm{mmol} / \mathrm{L}$ Tris- $\mathrm{HCl} \mathrm{pH} 8$, $150 \mathrm{mmol} / \mathrm{L} \mathrm{NaCl}, 1 \% \mathrm{NP}-40,0.5 \%$ sodium deoxycholate, and $0.1 \%$ SDS). Protein concentrations were measured using a Bio-Rad (Hercules, CA) protein assay kit. Western blotting was performed as described previously. 22,23 Antibodies and dilutions were as follows: $\beta$-actin (A5691; Sigma-Aldrich, St. Louis, MO) at 1:30,000, Bad (9292; Cell Signaling Technology, Danvers, MA) at 1:1000, phospho-Bad (9291; Cell Signaling Technology) at 1:1000, Bcl-X (AM05; Calbiochem, La Jolla, CA) at 1:1000, Cdk2 (sc-163; Santa Cruz Biotechnology, Santa Cruz, CA) at 1:500, Cdk4 (sc-260; Santa Cruz Biotechnology) at 1:500, Cdk6 (sc-177; Santa Cruz Biotechnology) at 1:500, cyclin D1 (sc-718; Santa Cruz Biotechnology) at 1:1000, cyclin E (sc-247; Santa Cruz Biotechnology) at 1:500, and $\beta$-hCG (MS-1065-P0; NeoMarkers, Fremont, CA) at 1:100, followed by secondary antibody incubation using sheep-anti-mouse-HRP (RPN4201; GE Healthcare, Little Chalfont, UK) or goat-anti-rabbit-HRP (RPN4301; GE Healthcare) at 1:3000. Films were developed from blots incubated using an Amersham ECL Plus kit (GE Healthcare, Piscataway, NJ).

\section{Analysis of Cell Proliferation}

To analyze cell proliferation, 2750 cells (T29 and T29 $\beta$-hCG) or 3500 cells (T80 and T80 $\beta$-hCG) were seeded onto 96-well plates in sets of $\mathrm{N}=8$ wells, including three control wells with medium only. Cells were incubated at $37^{\circ} \mathrm{C}$ and $5 \% \mathrm{CO}_{2}$. Proliferation was assessed at 24,48 , 60 , and 72 hours in triplicate, based on the colorimetric MTT cell proliferation assay (30-1010K; ATCC, Manassas, VA) according to the manufacturer's protocol.

\section{Soft Agarose Anchorage-Independence Assay}

To evaluate the influence of $\beta$-hCG overexpression on anchorage-independent colony formation in soft $\operatorname{agar}^{23}$ $5 \times 10^{4}$ T29, T80, T29 $\beta$-hCG, or T80 $\beta$-hCG cells of similar cell passage and age in culture were suspended in $2 \mathrm{~mL}$ medium with $0.35 \%$ low-melting agarose (Life Technologies, Carlsbad, CA) and the suspension was placed on top of $5 \mathrm{~mL}$ solidified $0.7 \%$ low-melting agarose. Triplicate cultures of each cell type were maintained for 14 days at $37^{\circ} \mathrm{C}$ in $5 \% \mathrm{CO}_{2}$; fresh $0.35 \%$ low-melting agarose with growth medium was added every 7 days. The assay was repeated three times. Colonies larger than $50 \mu \mathrm{m}$ in diameter, or containing more than 100 cells, 
were identified and counted at 14 to 20 days by microscopy under $\times 200$ magnification.

\section{Evaluation of Cell Cycle Progression and Apoptotic Status}

The cell cycle progression status of T29, T80, T29 $\beta$-hCG, and T80 $\beta$-hCG cell lines was evaluated after trypsinization, two washes in PBS, and resuspension at $1 \times 10^{6}$ to $2 \times 10^{6}$ cells in $200 \mu$ L PBS. Cells were fixed in cold $75 \%$ ethanol for a minimum of 4 hours at $4^{\circ} \mathrm{C}$, washed twice with PBS, resuspended in $200 \mu \mathrm{L}$ PBS, and incubated with $20 \mu \mathrm{L}$ of RNase ( $1 \mathrm{mg} / \mathrm{mL}$; Sigma-Aldrich) at $37^{\circ} \mathrm{C}$ for 15 to 20 minutes before staining with $200 \mu \mathrm{L}$ propidium iodide (50 $\mu \mathrm{g} / \mathrm{mL}$; Sigma-Aldrich). Determination of cell cycle progression was performed using a FACSCalibur flow cytometer (BD Biosciences, San Jose, CA) running BD CellQuest Pro version 5.1 software in the Flow Cytometry and Cellular Imaging Core Facility at the University of Texas M.D. Anderson Cancer Center (UTMDACC).

For apoptosis analysis, cells were harvested by trypsinization at 75\% confluence, washed twice with PBS, stained with fluorescein isothiocyanate-annexin $\mathrm{V}$ and propidium iodide (Apoptosis Detection Kit I; BD Biosciences), and analyzed using a FACSCalibur flow cytometer running BD CellQuest Pro version 5.1 software. The percentage of apoptotic cells was calculated based on the height of the M1 peak along the annexin $\mathrm{V}$ and propidium iodide biparametric cytogram, representing the combined early and late apoptotic cell populations among the total cell population analyzed. The experiment was performed in duplicate and repeated twice.

\section{Xenograft Athymic Nude Mouse Tumor Model and Analysis}

To determine the influence of $\beta$-hCG overexpression on xenograft tumor formation and growth in vivo, T29, T80, T29 $\beta$-hCG, and T80 $\beta$-hCG cell lines were harvested, washed twice with PBS, resuspended at $3 \times 10^{6}$ to $5 \times$ $10^{6}$ cells in $150 \mu \mathrm{L}$ PBS, and injected subcutaneously into 4- to 6-week-old Swiss nu-nu/Ncr athymic nude mouse hind flank (outbred mouse colony maintained by the Department of Experimental Radiation Oncology at UTMDACC). The mice were housed and cared for in the UTMDACC pathogen-free experimental animal facility and were checked every 2 days for tumors. The date on which the first grossly visible tumors appeared was recorded, and tumor size was monitored thereafter every 2 days. Mice were sacrificed after 13 weeks of follow-up, or according to tumor burden. Tumors were removed, fixed in $10 \%$ formalin, and subjected to routine histological examination. IHC staining for $\beta$-hCG (Santa Cruz Biotechnology; 1:1000 dilution), CD34 (Dako, Glostrup, Denmark; 1:100 dilution), and SV40 (sc-147; Santa Cruz Biotechnology; 1:50 dilution) was performed using avidin-biotinperoxidase methods, as described previously. ${ }^{21} \mathrm{IHC}$ staining for cytokeratins, estrogen receptor, and progesterone receptor was performed at the Histology and Tissue Processing Facility Core at UTMDACC. All mouse experiments were conducted according to our IACUCapproved animal protocol.

\section{IHC Analysis of $\beta$-hCG Expression in Human Gynecologic Tissues and Tumors}

We evaluated normal and cancerous ovarian tissues from previously designated tissue arrays, ${ }^{24}$ as well as normal fallopian tube and endometrium, according to our UTMDACC Institutional Review Board-approved protocol. Tissue arrays contained normal surface ovarian epithelium samples $(n=20)$, normal fallopian tube epithelium samples $(n=20)$, non-neoplastic cystic lesions $(n=$ $10)$, cystadenomas of ovary $(n=18)$, borderline ovarian tumors of low malignant potential $(n=14)$, and the eight major histotypes of ovarian epithelial carcinoma: high-grade serous ( $n=414)$, endometrioid $(n=48)$, mucinous $(n=7)$, clear cell $(n=13)$, malignant mixed mesodermal tumor of ovaries ( $n=13)$, undifferentiated $(n=10)$, transitional cell $(n=12)$, and low-grade serous $(n=22)$.

Immunohistochemical (IHC) staining for $\beta$-hCG was performed by using avidin-biotin-peroxidase methods on arrays of neoplastic, non-neoplastic, and normal human ovarian, fallopian tube, or endometrial tissue specimens, according to our previously published protocol. ${ }^{25}$ Briefly, tissue sections were deparaffinized, subjected to antigen retrieval, blocked, incubated overnight at $4^{\circ} \mathrm{C}$ with $\beta$-hCG antibody (MAB331; R\&D Systems, Minneapolis, MN; 1:100 dilution), incubated with biotinylated-goat-anti-rabbit for 30 minutes at room temperature, visualized with the chromogen 3,3'-diaminobenzidine, and lightly counterstained with hematoxylin. The primary antibody was replaced with PBS as negative control. $\beta$-hCG IHC staining integrity was independently verified and analyzed by two gynecologic pathologists (J.L. and F.Y.). Positive scoring (performed by F.Y. and S.Z.) was based on cytoplasmic staining of epithelial cells. The extent of $\beta$-hCG staining in tissue cores was quantified using a four-tier grading system: $0=<5 \%$ positive staining, $1=5 \%$ to $20 \%$ positive staining, $2=20 \%$ to $50 \%$ positive staining, and $3=>50 \%$ positive staining. For statistical analysis, we divided cases into two groups: negative expression (with scores of 0 ) and positive expression (with scores of 1,2 , or 3 ).

\section{Statistical Analysis}

Statistically significant differences in the rate of cell proliferation between cells with $\beta$-hCG expression and their parental control cells were calculated using linear, nonparametric regression analysis. Statistically significant differences in colony formation and tumor growth curves between cells with $\beta$-hCG expression and their parental control cells were calculated using Student's $t$-test. Fisher's exact test analysis was performed to evaluate the differences of $\beta$-hCG expression in normal ovarian specimens, non-neoplastic ovarian lesions, and malignant tumors, as well as $\beta$-hCG expression in different histological types of ovarian epithelial malignancies. All results 
were considered statistically significant at the $P<0.05$ confidence level. The SPSS version 17.02 software package was used (SPSS-IBM, Chicago, IL).

\section{Results}

\section{Overexpression of $\beta$-hCG in Immortalized Ovarian Surface Epithelial Cells Promotes Proliferation and Anchorage-Independent Colony Growth}

To determine the effect of elevated $\beta$-hCG expression on ovarian epithelial cell growth and transformation, we introduced a vector expressing $\beta$-hCG cDNA into immortalized nontumorigenic ovarian surface epithelial T29 and T80 cells by retrovirus infection, generating T29 $\beta$-hCG and T80 $\beta$-hCG cell lines. Western blot analysis demonstrated elevated expression of the $\beta$-hCG individual subunit, as well as the presence of $\beta$-hCG in the hCG heterodimeric complex, in both cell lines after infection with $\beta$-hCG CDNA (Figure 1A). Subsequently, we evaluated cell proliferation and anchorage-independent growth in soft agarose of T29 $\beta$-hCG and T80 $\beta$-hCG cells. T29 $\beta$-hCG showed a statistically significant increase in the rate of cell population doubling over the course of 24, 48, and 60 hours (slope $=0.0073 \pm 0.00044 \mathrm{AU} /$ hour), relative to parental T29 (slope $=0.005 \pm 0.00046 \mathrm{AU} /$ hour) $(P<0.0005$; Figure 1B), and T80 $\beta$-hCG cells showed a statistically significant increase at 24,48 , and 72 hours (slope $=0.0069 \pm 0.00057 \mathrm{AU} /$ hour), relative to parental T80 (slope $=0.0044 \pm 0.00057 \mathrm{AU} /$ hour $)(P<0.0005$; Figure 1C). Furthermore, T29 $\beta$-hCG and T80 $\beta$-hCG cells displayed increased anchorage-independent growth, relative to parental T29 and T80 cells $(P<0.01$ and $P=$ 0.01 , respectively; Figure $1 D)$. These data indicate that $\beta$-hCG overexpression promotes cell proliferation and anchorage-independent growth of ovarian epithelial cells in vitro.

\section{$\beta$-hCG Overexpression Induces Cell Cycle Progression through Elevated Expression of Cyclins and Cdk Activation Partners}

Next, we sought to determine the effect of overexpression of $\beta$-hCG on cell cycle status in ovarian epithelial cells. Flow cytometry analysis of cell cycle progression revealed decreased G1 cell populations in T29 $\beta$-hCG cells (48.8\% versus $39.5 \%$ ) and T80 $\beta$-hCG cells $(52.5 \%$ versus $43.1 \%$ ), as well as increased G2 cell populations in T29 $\beta$-hCG cells (22.6\% versus $33.3 \%$ ) and T80 $\beta$-hCG cells $(19.7 \%$ versus $29.8 \%$ ), relative to controls (Figure $2 \mathrm{~A})$. Furthermore, Western blot analysis revealed that overexpression of $\beta$-hCG led to up-regulation of cyclins $\mathrm{E}$ and D1 and their associated partner cyclin-dependent kinases Cdk2 and Cdk4, as well as Cdk6, relative to parental controls (Figure 2B). These data suggest that $\beta$-hCG expression potentiates proliferation and in vitro tumor transformation of ovarian surface epithelial cells by promoting progression through the G2-checkpoint of the
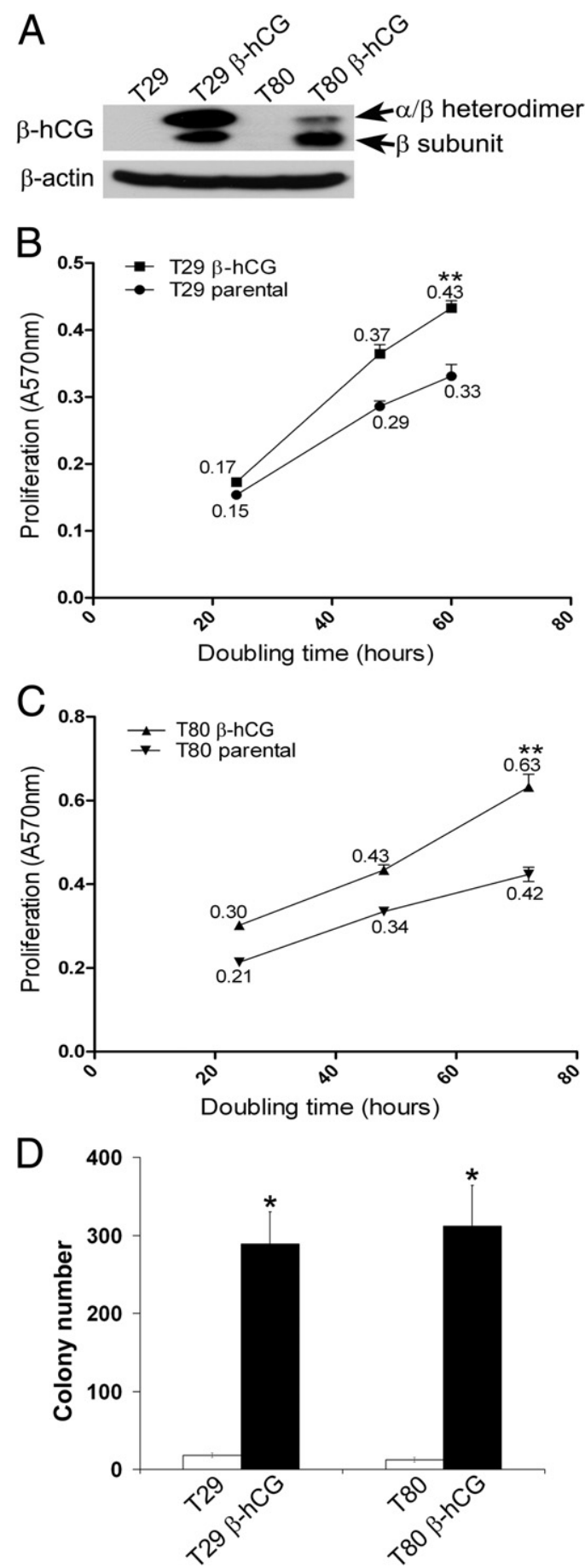

Figure 1. Expression of $\beta$-hCG in immortalized ovarian surface epithelial cells promotes cell proliferation and anchorage-independent growth. $\mathbf{A}$ Western analysis revealed elevated expression of the $\beta$-hCG subunit alone, as well as the $\beta$-subunit in heterodimeric complex with the $\alpha$-subunit, after $\beta$-hCG cDNA infection of immortalized ovarian surface epithelial T29 and T80 cells. B: T29 $\beta$-hCG showed a statistically significant increase in the rate of cell proliferation over the course of 24,48 , and 60 hours (slope $=0.0073 \pm 0.00044 \mathrm{AU} /$ hour), relative to similar-passage parental T29 (slope $=0.005 \pm 0.00046 \mathrm{AU} /$ hour). Numeric values indicate proliferation rate. AU, arbitrary units. ${ }^{* *} P<0.0005$. C: T80 $\beta$-hCG cells similarly showed a statistically significant increase at 24,48 , and 72 hours (slope $=0.0069 \pm 0.00057 \mathrm{AU} /$ hour), relative to similar-passage parental T80 cells (slope $=0.0044 \pm 0.00057 \mathrm{AU} /$ hour). Numeric values indicate proliferation rate. ${ }^{* * *} P<0.0005$. D: T29 $\beta$-hCG and T80 $\beta$-hCG cells showed a statistically significant increase in anchorage-independent colony formation, compared with similar-passage parental T29 and T80 cells. Error bars indicate mean $\pm \mathrm{SD} .{ }^{*} P \leq 0.01 ; n=3$. 
A
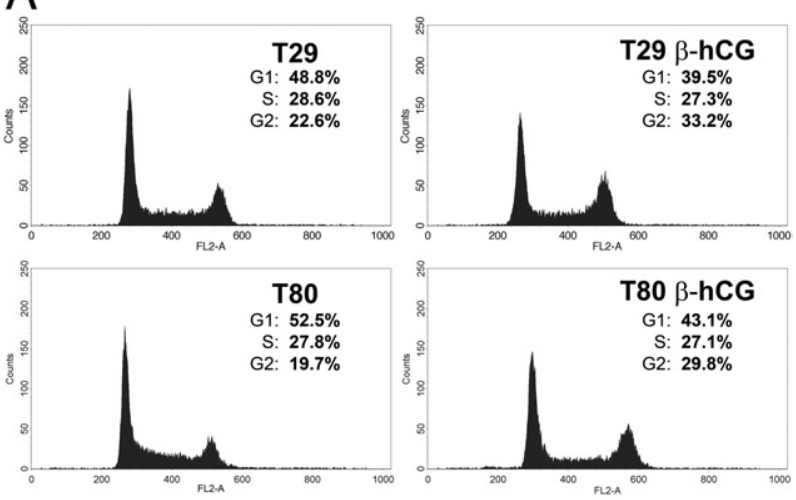

B

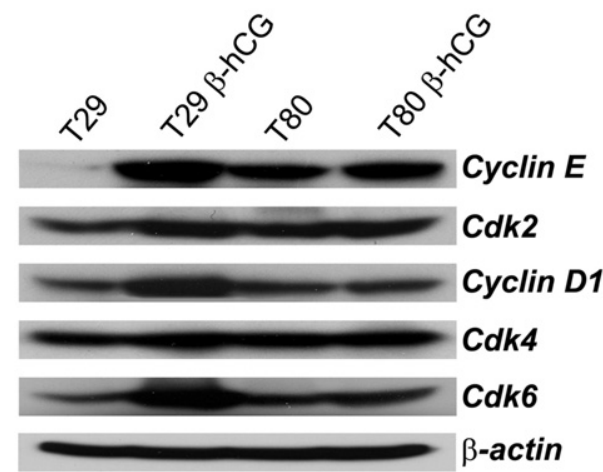

Figure 2. $\beta$-hCG expression promotes cell cycle progression and up-regulation of cyclins and associated cyclin-dependent kinases in immortalized ovarian surface epithelial cells. A: Flow cytometry analysis of cell cycle progression revealed decreased G1 cell populations in T29 $\beta$-hCG cells ( $48.8 \%$ versus $39.5 \%$ ) and T80 $\beta$-hCG cells $(52.5 \%$ versus $43.1 \%$ ), as well as increased G2-phase in T29 $\beta$-hCG cells (22.6\% versus 33.2\%) and T80 $\beta$-hCG cells $(19.7 \%$ versus $29.8 \%$ ), relative to T29 or T80 parental control cells. B: Up-regulation of cyclins E and D1, as well as of Cdk2, Cdk4, and Cdk6, was demonstrated in T29 $\beta$-hCG and T80 $\beta$-hCG cells, relative to parental control cells.

cell cycle and by regulation of cell cycle proteins cyclin $\mathrm{E}$, cyclin D1, and Cdk2, Cdk4, and Cdk6 to coordinately regulate progression of the cell cycle.

\section{$\beta$-hCG Overexpression Attenuates Apoptosis via Elevated Prosurvival and Decreased \\ Prodeath Proteins}

We next evaluated the effect of $\beta$-hCG on apoptotic status. Flow cytometry analysis of apoptotic index revealed a significant decrease in T29 $\beta$-hCG cells $(2.9 \%$ versus $12.3 \% ; P<0.05)$ and T80 $\beta$-hCG cells $(3.1 \%$ versus 9.2\%; $P<0.05$ ), relative to parental control cell lines (Figure 3A). Western blot analysis revealed that overexpression of $\beta$-hCG led to up-regulation of the antiapoptotic, prosurvival protein $\mathrm{BCl}-\mathrm{X}_{\mathrm{L}}$, as well as a significant decrease in the active form of proapoptotic protein Bad, without altering total Bad protein (Figure 3B). These data indicate that elevated $\beta$-hCG expression decreases the overall tendency toward apoptosis in ovarian surface epithelial cells, further facilitating the process of cell transformation.

\section{$\beta$-hCG Overexpression Transforms}

Immortalized Ovarian Surface Epithelial Cells Resulting in Enhanced Xenograft Tumorigenesis

Next, we investigated the effect of elevated $\beta$-hCG on tumor growth in vivo. T29, T80, T29 $\beta$-hCG, and T80 $\beta$-hCG cells were injected as subcutaneous xenografts into athymic nude mice. Tumor incidence was recorded as 13 of 24 T29 $\beta$-hCG cell injections in 10 mice and in 6 of 14 T80 $\beta$-hCG cell injections in 7 mice (Figure $4, \mathrm{~A}$ and C); no tumor growth was observed in the 4 mice injected with T29 or T80 parental control cells after 13 weeks of observation (Figure 4, A and C). A histopathological analysis of xenograft tumors produced by the injection of T29 $\beta$-hCG or T80 $\beta$-hCG cells revealed poorly differentiated carcinoma (Figure 4, B and D). IHC analysis of representative T80 $\beta$-hCG-derived tumors identified an intense epithelial membrane and cytoplasmic $\beta$-hCG immunoreactivity pattern with extracellular secretion (Figure 4E), positive reactivity for SV4O T/t antigens indicating tumor formation due to immortalized human cells (Figure 4F), a high density of CD34-positive microvessels (Figure 4G),
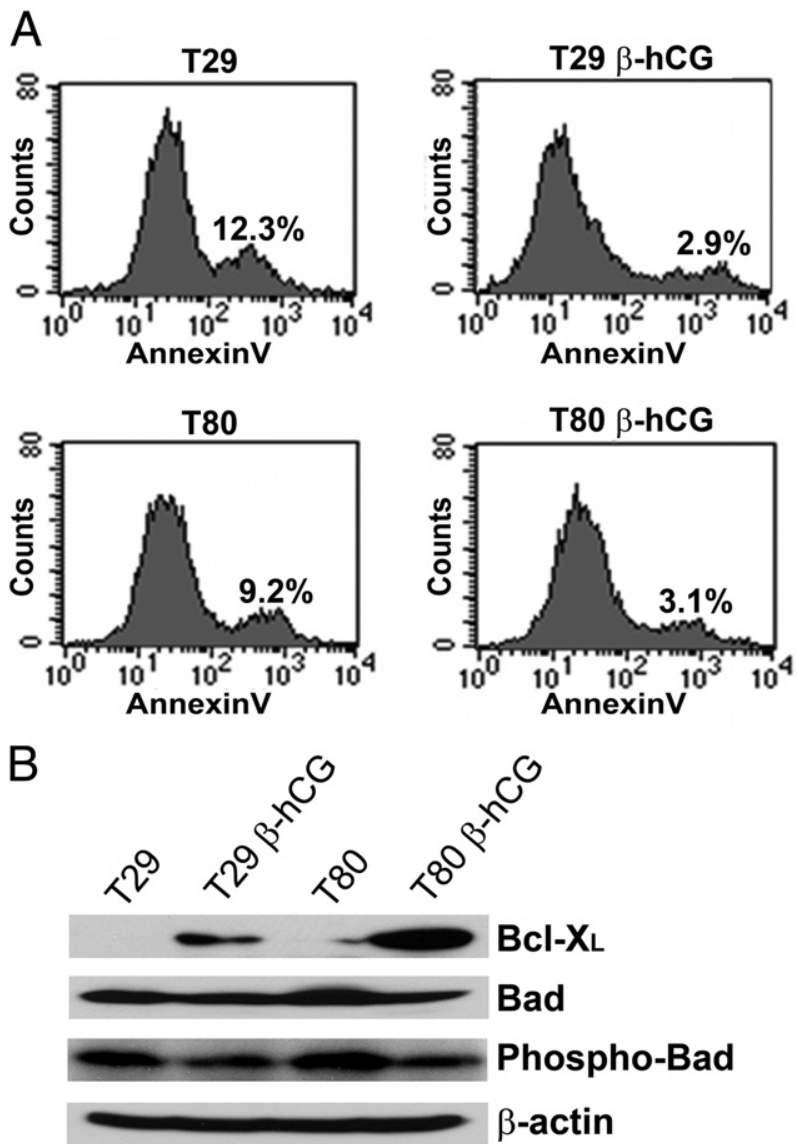

Figure 3. $\beta$-hCG expression attenuates apoptosis, up-regulates progrowth $\mathrm{Bcl}-\mathrm{X}_{\mathrm{L}}$, and down-regulates proapoptotic phospho-Bad in immortalized ovarian surface epithelial cells. A: Apoptosis was markedly decreased in T29 $\beta$-hCG cells relative to T29 parental cells $(2.9 \%$ versus $12.3 \%, P<0.05)$, as well as in T80 $\beta$-hCG cells relative to T80 parental cells (3.1\% versus $9.2 \%$, $P<0.05)$. B: Up-regulation of the prosurvival, antiapoptotic protein $\mathrm{Bcl}-\mathrm{X}_{\mathrm{L}}$, with a concurrent decrease in the proapoptotic phospho-Bad protein, was demonstrated in T29 $\beta$-hCG and T80 $\beta$-hCG cells, relative to parental control cells 
1390 Guo et al

AJP September 2011, Vol. 179, No. 3

strong cytoplasmic cytokeratin reactivity indicative of epithelial lineage (Figure $4 \mathrm{H}$ ), positive nuclear progesterone receptor immunostaining (Figure $4 \mathrm{I}$ ), and negative immunoreactivity for nuclear estrogen receptor (Figure 4J). Collectively, these data demonstrate that the $\beta$-hCG-mediated cell cycle progression, increased proliferation, and decreased apoptosis yield a transformation of im-
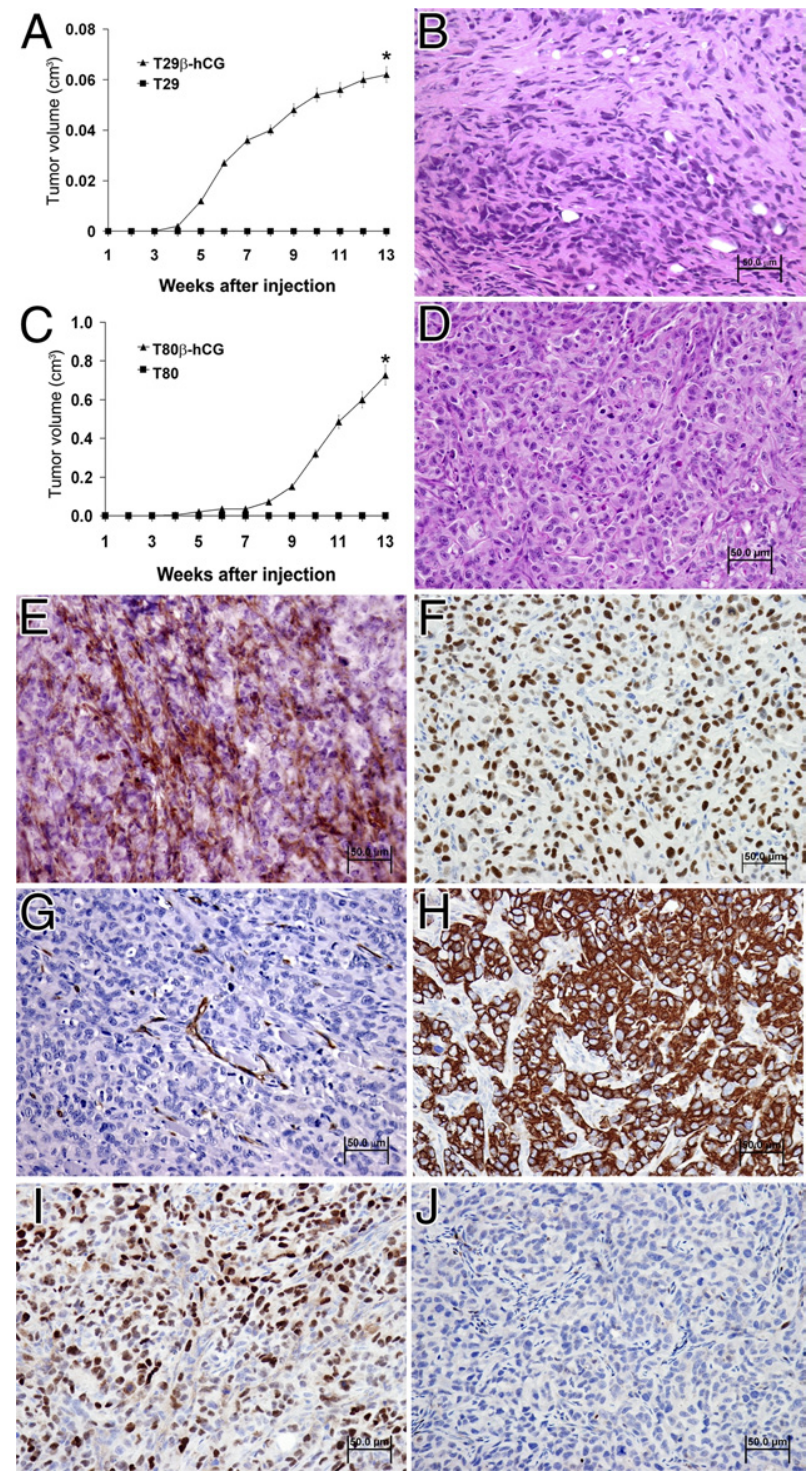

Figure 4. $\beta$-hCG expression results in transformation of immortalized ovarian surface epithelial cells. A and $\mathbf{C}$ : Growth rate of subcutaneous xenografts in nude mice inoculated with T29 $\beta$-hCG and T80 $\beta$-hCG cells, respectively: 13 of 24 injections in 10 mice grew tumors using T29 $\beta$-hCG cells, and 6 of 14 injections in 7 mice using T80 $\beta$-hCG cells, compared with an incidence of 0 tumors in 4 mice injected with T29 or T80 parental control cells, after 13 weeks of observation (mean $\pm \mathrm{SD}$ ). ${ }^{*} P<0.01$. B and D: H\&E staining of subcutaneous xenograft tumors produced by injection of T29 $\beta$-hCG and T80 $\beta$-hCG cells, respectively, revealed poorly differentiated carcinoma morphology. E-J: IHC staining of representative T80 $\beta$-hCG xenograft tumor sections, in which the intensity of brown deposition represents marker expression highlighted by blue/purple hematoxylin counterstaining, demonstrates membrane and cytoplasmic $\beta$-hCG reactivity $(\mathbf{E})$, reactivity of SV40 T/t antigens indicating tumor formation by human cells $(\mathbf{F})$, CD34 reactivity in recruited microvessels (G), cytoplasmic cytokeratin reactivity indicating epithelial cell lineage $(\mathbf{H})$, nuclear progesterone receptor reactivity $(\mathbf{I})$, and negative estrogen receptor reactivity $(\mathbf{J})$.
Table 1. $\beta$-hCG IHC Expression in Normal Ovarian and Fallopian Tube Epithelium Compared with that in Benign, Borderline, and Malignant Ovarian Lesions

\begin{tabular}{lccc}
\hline \multicolumn{1}{c}{ Tissue or tumor type } & $n$ & $\begin{array}{c}\text { Positive } \\
(\%)\end{array}$ & $\begin{array}{c}P \\
\text { value }^{*}\end{array}$ \\
\hline $\begin{array}{l}\text { Normal fallopian tube } \\
\quad \text { epithelium }\end{array}$ & 20 & 0 & \\
$\quad$ Normal ovarian epithelium & 20 & 0 & \\
Benign cyst & 10 & 20 & $>0.05$ \\
Cystadenoma & 18 & 22 & $<0.05$ \\
Low malignant potential & 14 & 71 & $<0.001$ \\
Low-grade serous carcinoma & 22 & 33 & $<0.05$ \\
High-grade serous carcinoma & 414 & 37 & $<0.001$ \\
Total & 518 & & \\
\hline
\end{tabular}

${ }^{*}$ Compared with normal ovarian and normal fallopian tube epithelium

mortalized ovarian surface epithelial cells, facilitating tumor growth.

\section{$\beta$-hCG Expression Is Elevated in Human Ovarian Tumor Tissue Relative to Normal Ovarian, Fallopian Tube, and Endometrial Epithelium}

In light of our in vitro and in vivo data showing that $\beta$-hCG functionally promotes tumorigenic behavior, we sought to determine the immunoreactivity pattern in human ovarian cancer. A survey of $\beta$-hCG expression was conducted using 518 tissue samples from normal ovary, fallopian tube, and endometrium, as well as from all ovarian tumor histological types. $\beta$-hCG immunoreactivity, detected as diffuse cytoplasmic staining with moderate to high intensity in varying proportions of epithelial cells, was significantly higher in high-grade, low-grade, and borderline tumors of low malignant potential (all $P<0.01$ ), compared with normal ovarian surface epithelium, falIopian tube epithelium, and endometrium, as well as benign ovarian cystic lesions (Table 1). However, $\beta$-hCG expression did not significantly vary among the eight major ovarian carcinoma histotypes in 539 patient samples (Table 2). Of note, variation in $\beta$-hCG expression was not a significant independent predictor of overall survival or disease-free survival among the

Table 2. $\beta$-hCG Expression in Different Histologic Types of Ovarian Epithelial Malignancies

\begin{tabular}{lrcc}
\hline \multicolumn{1}{c}{ Tissue or tumor type } & $n$ & $\begin{array}{c}\text { Positive } \\
(\%)\end{array}$ & $\begin{array}{c}P \\
\text { value* }^{*}\end{array}$ \\
\hline High-grade serous & 414 & 37 & \\
$\quad$ carcinoma & & & \\
Endometrioid carcinoma & 48 & 35 & \\
Mucinous carcinoma & 7 & 29 & \\
$\quad$ Clear cell carcinoma & 13 & 46 & \\
Malignant mixed mesodermal & 13 & 31 & \\
$\quad$ tumors & & & \\
Undifferentiated carcinoma & 10 & 50 & \\
Transitional cell carcinoma & 12 & 17 & \\
Low grade serous carcinoma & 22 & 32 & 0.8 \\
$\quad$ Total & 539 & & \\
\hline
\end{tabular}

${ }^{*}$ Fisher's exact test. 


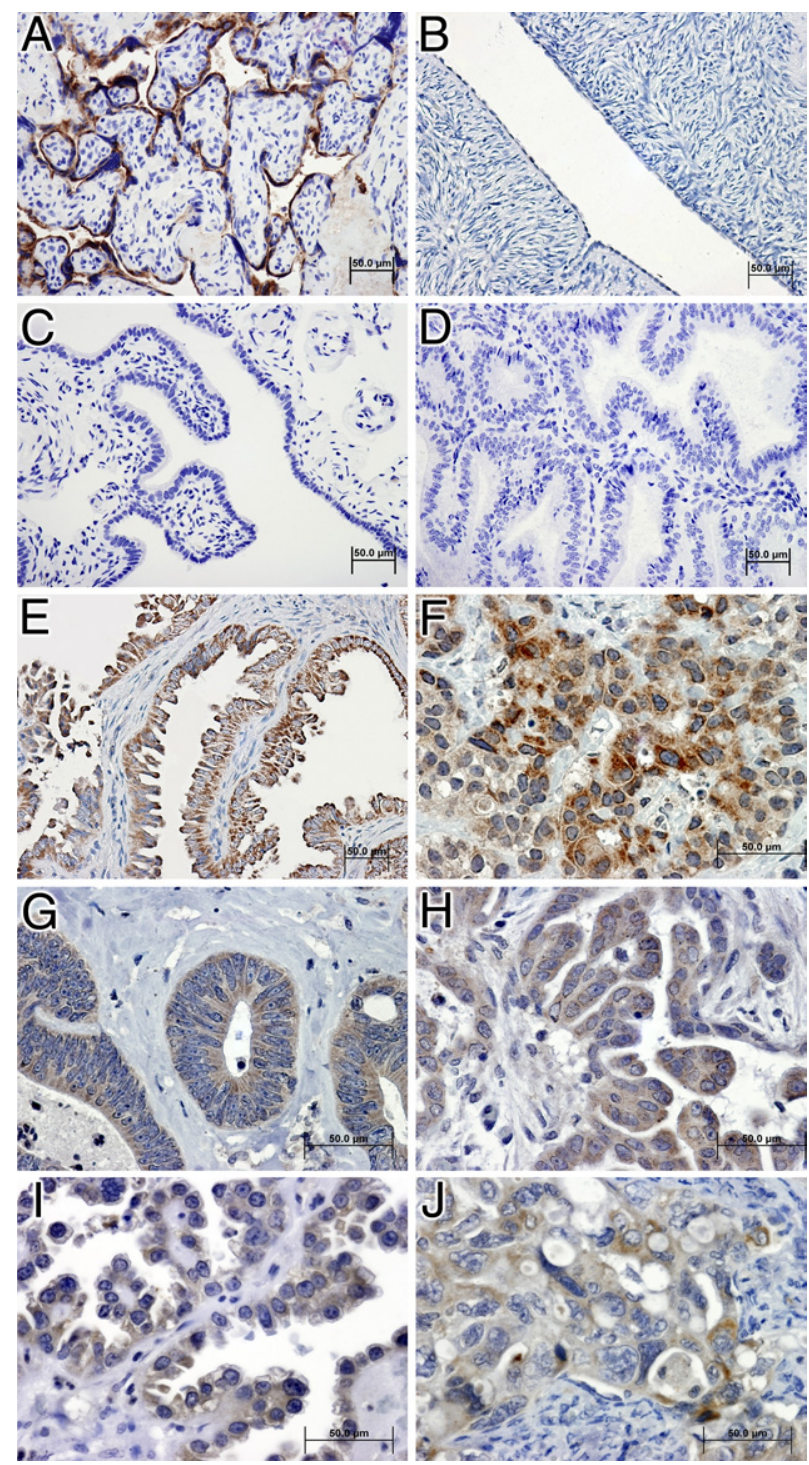

Figure 5. $\beta$-hCG immunoreactivity is highly elevated in human ovarian neoplastic and malignant carcinoma ovarian cancer patients, compared with normal tissue. Representative tissue sections, in which the intensity of brown deposition represents $\beta$-hCG expression highlighted by blue/ purple hematoxylin counterstaining, depicts characteristic $\beta$-hCG immunoreactivity in positive-control placental epithelium (A), negative normal ovarian epithelium (B), negative normal fimbriated fallopian tube epithelium (C), negative endometrium (D), positive low malignant potential ovarian tumor (E), strongly positive high-grade serous carcinoma (F), positive endometrioid carcinoma (G), positive low-grade serous carcinoma (H), positive clear cell carcinoma (I), and positive mucinous carcinoma $(\mathbf{J})$. Scale bars $=50 \mu \mathrm{m}$. Original magnification: $\times 200(\mathbf{A}-\mathbf{E})$; $\times 400(\mathbf{F}-\mathbf{J})$

major ovarian cancer histotypes (data not shown). These data suggest that $\beta$-hCG expression alone may not be a distinguishing factor for histotype-specific malignancy or clinical outcome in ovarian carcinoma. Representative $\beta$-hCG immunostaining patterns are shown in Figure 5 for placenta as a positive control (Figure 5A), negative epithelial staining in normal ovarian tissue (Figure 5B), fimbriated fallopian tube (Figure $5 C$ ), and endometrium (Figure 5D), and positive epithelial staining in tissue with low malignant potential (Figure 5E), as well as positive staining in high-grade serous carcinoma (Figure 5F), endometrioid carcinoma (Figure 5G), low-grade serous carcinoma (Figure 5H), clear cell carcinoma (Figure 5I), and mucinous carcinoma (Figure 5J).

\section{Discussion}

With the present study, we demonstrate for the first time that the hormone-regulated $\beta$-hCG heterodimer subunit functions as a tumor promoting factor in human ovarian epithelial cells, indicating that $\beta$-hCG may play a role in the etiology and development of human ovarian carcinoma. Our transformation studies using in vivo mouse subcutaneous xenografts established that immortalized T29 and T80 ovarian epithelial cell lines overexpressing $\beta$-hCG display markedly increased tumor formation and growth, compared with tumor-free control T29 and T80 xenografts. Strikingly, in vitro tumorigenic mechanistic analysis revealed that T29 $\beta$-hCG and T80 $\beta$-hCG cell lines showed increased cell cycle progression through the $\mathrm{G} 2$ checkpoint and elevated expression of cyclin E, cyclin D1, Cdk2, Cdk4, and Cdk6, compared with control T29 and T80 lines. Moreover, T29 $\beta$-hCG and T80 $\beta$-hCG displayed a reduced apoptotic index, with up-regulated expression of the prosurvival, antiapoptotic protein Bcl$X_{L}$, and attenuation of the phosphorylation level of one of the proapoptotic proteins, Bad, compared with control T29 and T80 lines. Furthermore, T29 $\beta$-hCG and T80 $\beta$-hCG cell lines both displayed an elevated resistance to the relatively higher rate of morphological senescence observed in parental T29 and T80 cells (data not shown). Thus, our data strongly indicate that acquisition of $\beta$-hCG expression results in an enhanced prosurvival, antiapoptotic, and cell cycle-promoting status, collectively facilitating in vivo tumorigenesis.

Additionally, elevated $\beta$-hCG expression was observed in all human ovarian tumor histotypes, compared with largely negative expression observed in normal ovarian, fallopian tube, and endometrium epithelial cells. According to recent reports, the tissue of origin for ovarian carcinoma may be the surface epithelium of the ovaries or epithelial cells of the fimbriated end of the fallopian tube. ${ }^{20}$ Our data indicate that neither of these normal tissues expresses high levels of $\beta$-hCG, suggesting that regulation may not be tissue-type specific. Further, it is of interest that we observed elevated $\beta$-hCG in a high percentage of tumors diagnosed as low malignant potential. These low malignant potential tumors are predicted to follow one of two distinct pathways of progression: either they recur as tumors maintaining classification as low malignant potential or they present after several years as a low-grade serous carcinoma. ${ }^{20}$ Our in vitro and in vivo data suggest that $\beta$-hCG may play an important role in the signaling mechanisms promoting malignant transformation along the pathway toward development of lowgrade serous carcinoma.

Recent studies have addressed the expression profile of $\beta$-hCG in ovarian cancer patients. Nowak-Markwitz et $\mathrm{al}^{19}$ reported elevated transcription and distribution of $\beta$-hCG in eight cases of ovarian epithelial carcinoma. 
Jankowska et al ${ }^{9}$ found elevated transcription and coexpression of $\beta$-hCG and its receptor, LH/hCGR, in nontrophoblastic ovarian, endometrial, uterine, and cervical cancers, suggesting that coexpression of $\beta$-hCG and LH/hCGR mediates an autocrine/paracrine regulation loop controlling nontrophoblastic tumor growth. Our present results confirm and expand on these findings, identifying cell cycle progression and attenuation of apoptosis as specific, $\beta$-hCG-mediated mechanisms promoting tumorigenic potential in ovarian cancer epithelial cells.

More work remains to be done in fully elucidating the detailed molecular mechanisms of $\beta$-hCG-mediated tumorigenesis in ovarian cancer. $\beta$-hCG promotes mitogenic activation of human breast cells, which may contribute to malignant transformation. ${ }^{26}$ Silencing $\beta$-hCG expression in human cervical carcinoma cells promoted apoptosis, suggesting that $\beta$-hCG might mediate a similar antiapoptotic mechanism in cervical cancer. ${ }^{18}$ Abnormal Wnt gene expression was linked to the development of malignant breast lobuloalveolar growth in a transgenic mouse model of $\beta$-hCG overexpression, suggesting that dysregulated Wht signaling may elevate $\beta$-hCG expression, promoting development of breast carcinoma. ${ }^{25} \mathrm{Fi}-$ nally, pUbiC-hCG $\beta_{\sqrt{5}}$ transgenic mice displayed an abnormal ovarian function that exerted a dominant regulatory control over the development of both pituitary adenoma and lobuloalveolar mammary adenocarcinoma, ${ }^{27}$ suggesting that ovarian elevated $\beta$-hCG may play an extrinsic role in modulating tumorigenic potential. Coupled with our present observations, these recent findings highlight the critical functional role of $\beta$-hCG in nontrophoblastic gynecologic malignancies. There is a possibility that hCG fragments detected in human tumors do not have the same activity as the full-length $\beta$-hCG sequence expressed in our in vitro and in vivo study, and therefore the exact relevance of our studies to human ovarian cancer cell hCG fragment expression remains unknown. Future studies are needed to better define the underlying molecular mechanisms of $\beta$-hCG-induced ovarian tumorigenesis.

In summary, our data demonstrate that $\beta$-hCG can induce transformation of ovarian epithelial cells by increasing cell cycle progression and attenuating apoptosis to promote tumorigenic initiation and growth. We believe that uncontrolled expression of $\beta$-hCG has a potential etiological role in ovarian carcinogenesis. In combination with findings from other recent studies, our results shed light on the molecular mechanisms of $\beta$-hCG expression and its functional role in promoting ovarian carcinoma and thus may point to a new target for therapeutic intervention.

\section{Acknowledgments}

We thank the members of the Liu laboratory for helpful discussions and technical assistance, and Wendy Schober, MLT, for her assistance with flow cytometry analysis.

\section{References}

1. Jemal A, Siegel R, Ward E, Hao Y, Xu J, Thun MJ: Cancer statistics, 2009. CA Cancer J Clin 2009, 59:225-249

2. Park JT, Li M, Nakayama K, Mao TL, Davidson B, Zhang Z, Kurman RJ, Eberhart CG, Shih le M, Wang TL: Notch3 gene amplification in ovarian cancer. Cancer Res 2006, 66:6312-6318

3. Cho KR, Shih le M: Ovarian cancer. Annu Rev Pathol 2009, 4:287-313

4. Auner V, Kriegshäuser G, Tong D, Horvat R, Reinthaller A, Mustea A, Zeillinger R: KRAS mutation analysis in ovarian samples using a high sensitivity biochip assay. BMC Cancer 2009, 9:111

5. Dehari R, Kurman RJ, Logani S, Shih leM: The development of highgrade serous carcinoma from atypical proliferative (borderline) serous tumors and low-grade micropapillary serous carcinoma: a morphologic and molecular genetic analysis. Am J Surg Pathol 2007, 31:1007-1012

6. Pierce JG, Parsons TF: Glycoprotein hormones: structure and function. Annu Rev Biochem 1981, 50:465-495

7. Ozturk M: Human chorionic gonadotropin, its free subunits and gestational trophoblastic disease. J Reprod Med 1991, 36:21-26

8. Bagshawe KD: Choriocarcinoma: a model for tumour markers. Acta Oncol 1992, 31:99-106

9. Jankowska A, Andrusiewicz M, Grabowski J, Nowak-Markwitz E, Warchol JB: Coexpression of human chorionic gonadotropin beta subunit and its receptor in nontrophoblastic gynecological cancer. Int J Gynecol Cancer 2008, 18:1102-1107

10. Nowak-Markwitz E, Jankowska A, Andrusiewicz M, Szczerba A: Expression of beta-human chorionic gonadotropin in ovarian cancer tissue. Eur J Gynaecol Oncol 2004, 25:465-469

11. Carpelan-Holmström M, Haglund $\mathrm{C}$, Lundin J, Alfthan $\mathrm{H}$, Stenman $\mathrm{UH}$, Roberts PJ: Independent prognostic value of preoperative serum markers CA 242, specific tissue polypeptide antigen and human chorionic gonadotrophin beta, but not of carcinoembryonic antigen or tissue polypeptide antigen in colorectal cancer. Br J Cancer 1996, 74:925-929

12. Grossmann M, Hoermann R, Gocze PM, Ott M, Berger P, Mann K: Measurement of human chorionic gonadotropin-related immunoreactivity in serum, ascites and tumour cysts of patients with gynaecologic malignancies. Eur J Clin Invest 1995, 25:867-873

13. Grossmann M, Trautmann ME, Poertl S, Hoermann R, Berger $P$, Arnold R, Mann K: Alpha-subunit and human chorionic gonadotropinbeta immunoreactivity in patients with malignant endocrine gastroenteropancreatic tumours. Eur J Clin Invest 1994, 24:131-136

14. McLoughlin J, Pepera T, Bridger J, Williams G: Serum and urinary levels of beta human chorionic gonadotrophin in patients with transitional cell carcinoma. Br J Cancer 1991, 63:822-824

15. Szturmowicz M, Wiatr E, Sakowicz A, Slodkowska J, Roszkowski K, Filipecki S, Rowinska-Zakrzewska ER: The role of human chorionic gonadotropin beta subunit elevation in small-cell lung cancer patients. J Cancer Res Clin Oncol 1995, 121:309-312

16. Iles RK, Persad R, Trivedi M, Sharma KB, Dickinson A, Smith P, Chard $\mathrm{T}$ : Urinary concentration of human chorionic gonadotrophin and its fragments as a prognostic marker in bladder cancer. Br J Urol 1996, $77: 61-69$

17. Gillott DJ, Iles RK, Chard T: The effects of beta-human chorionic gonadotrophin on the in vitro growth of bladder cancer cell lines. $\mathrm{Br} \mathrm{J}$ Cancer 1996, 73:323-326

18. Jankowska A, Gunderson SI, Andrusiewicz M, Burczynska B, Szczerba A, Jarmolowski A, Nowak-Markwitz E, Warchol JB: Reduction of human chorionic gonadotropin beta subunit expression by modified U1 snRNA caused apoptosis in cervical cancer cells. Mol Cancer 2008, 7:26

19. Nowak-Markwitz E, Jankowska A, Szczerba A, Andrusiewicz M, Warchoł JB: Localization of human chorionic gonadotropin beta subunit transcripts in ovarian cancer tissue. Folia Histochem Cytobiol 2004, 42:123-126

20. Kurman RJ, Shih IeM: The origin and pathogenesis of epithelial ovarian cancer: a proposed unifying theory. Am J Surg Pathol 2010 34:433-443

21. Liu J, Yang G, Thompson-Lanza JA, Glassman A, Hayes K, Patterson A, Marquez RT, Auersperg N, Yu Y, Hahn WC, Mills GB, Bast RC Jr: A genetically defined model for human ovarian cancer. Cancer Res 2004, 64:1655-1663

22. Yang G, Cai KQ, Thompson-Lanza JA, Bast RC Jr, Liu J: Inhibition of breast and ovarian tumor growth through multiple signaling pathways 
by using retrovirus-mediated small interfering RNA against Her-2/neu gene expression. J Biol Chem 2004, 279:4339-4345

23. Yang G, Thompson JA, Fang B, Liu J: Silencing of H-ras gene expression by retrovirus-mediated siRNA decreases transformation efficiency and tumorgrowth in a model of human ovarian cancer. Oncogene 2003, 22:5694-5701

24. Rosen DG, Yang G, Deavers MT, Malpica A, Kavanagh JJ, Mills GB, Liu J: Cyclin E expression is correlated with tumor progression and predicts a poor prognosis in patients with ovarian carcinoma. Cancer 2006, 106:1925-1932

25. Kuorelahti A, Rulli S, Huhtaniemi I, Poutanen M: Human chorionic gonadotropin ( $\mathrm{hCG}$ ) up-regulates wnt5b and wnt7b in the mammary gland, and hCGbeta transgenic female mice present with mammary gland tumors exhibiting characteristics of the Wnt/beta-catenin pathway activation. Endocrinology 2007, 148:3694-3703

26. Hudelist G, Wuelfing P, Czerwenka K, Knofler M, Haider S, Fink Retter A, Gschwantler-Kaulich D, Pfeiler G, Kubista E, Singer CF Beta-hCG/LH receptor (b-hCG/LH-R) expression is increased in invasive versus preinvasive breast cancer: implications for breast carcinogenesis? J Cancer Res Clin Oncol 2009, 135:191-195

27. Rulli SB, Kuorelahti A, Karaer O, Pelliniemi LJ, Poutanen M, Huhtaniemi I: Reproductive disturbances, pituitary lactotrope adenomas, and mammary gland tumors in transgenic female mice producing high levels of human chorionic gonadotropin. Endocrinology 2002, 143:4084-4095 